\title{
A Preprocessing Algorithm for MIQP solvers with Applications to MPC
}

\author{
Daniel Axehill, Anders Hansson \\ Division of Automatic Control \\ Department of Electrical Engineering \\ Linköpings universitet, SE-581 83 Linköping, Sweden \\ WWW: http://Www. control.isy.liu.se \\ E-mail: daniel@isy.liu.se, hansson@isy.liu.se
}

29th April 2004

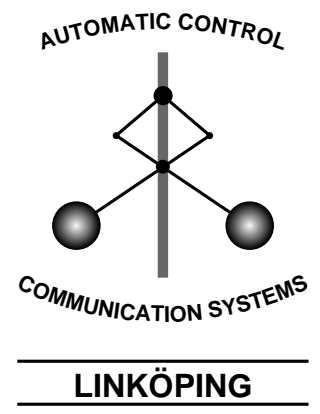

Report no.: LiTH-ISY-R-2607

Submitted to Reglermöte 2004

Technical reports from the Control \& Communication group in Linköping are available at http://www. control.isy.liu.se/publications. 


\begin{abstract}
In this paper a preprocessing algorithm for unconstrained mixed integer quadratic programming problems and binary quadratic programming problems is presented. The algorithm applies to problems with certain properties, which are further described in the paper. When the algorithm is applied to a problem with these properties, the optimal value for some or all integer variables can be computed without approximations in polynomial time. The algorithm is first derived for the binary quadratic programming problem and the result is then extended to the mixed integer quadratic programming problem by transforming the latter problem into the first problem. Both mentioned quadratic programming problems have several important applications. In this paper, the focus is on model predictive control problems with both real-valued and binary control signals. As an illustration of the method, the algorithm is applied to two different problems of this type.
\end{abstract}

Keywords: Predictive control, Integer programming, Quadratic programming 


\title{
A PREPROCESSING ALGORITHM FOR MIQP SOLVERS WITH APPLICATIONS TO MPC
}

\author{
Daniel Axehill * Anders Hansson* \\ ${ }^{*}$ Division of Automatic Control and Communication Systems \\ Department of Electrical Engineering, Linköpings universitet \\ SE-581 83 LINKÖPING, SWEDEN \\ \{daniel, hansson\} Cisy.liu.se
}

\begin{abstract}
In this paper a preprocessing algorithm for unconstrained mixed integer quadratic programming problems and binary quadratic programming problems is presented. The algorithm applies to problems with certain properties, which are further described in the paper. When the algorithm is applied to a problem with these properties, the optimal value for some or all integer variables can be computed without approximations in polynomial time. The algorithm is first derived for the binary quadratic programming problem and the result is then extended to the mixed integer quadratic programming problem by transforming the latter problem into the first problem. Both mentioned quadratic programming problems have several important applications. In this paper, the focus is on model predictive control problems with both real-valued and binary control signals. As an illustration of the method, the algorithm is applied to two different problems of this type.
\end{abstract}

Keywords: Predictive control, Integer programming, Quadratic programming

\section{INTRODUCTION}

An extension to ordinary Model Predictive Control (MPC) is to allow binary control signals, see for example (Bemporad and Morari, 1999). When this generalization is made, the ordinary Quadratic Programming (QP) solver has to be replaced with a so-called Mixed Integer Quadratic Programming (MIQP) solver, which also is able to optimize binary variables. Except for problems with particular structures, the MIQP problem is known to be $\mathscr{N} \mathscr{P}$-complete. This means that in worst case the solution time grows exponentially with the size of the problem (Bemporad et al., 1999). One way of reducing the average complexity is to use an algorithm which in most cases can find the global optimum without enumerating all possible solutions. A popular algorithm to use when solving MIQP problems is the branch and bound algorithm. In this report we have investigated if the performance of the branch and bound algorithm can be increased by using preprocessing. Only MIQP problems without constraints are considered. Previous work in the area of preprocessing for the MIQP problem is found in, e.g., (Savelsbergh, 1994).

In the first part of the derivation of the main result, a so-called Binary Quadratic Programming (BQP) problem is studied. The BQP problem is known to be $\mathscr{N P}$-hard, (Katayama and Narihisa, 2001). Most algorithms for this kind of problems either focus on producing approximative solutions or on only handling various special cases of the general problem, (Garey and Johnson, 1979). The algorithm presented in this paper belongs to the latter type of algorithms. Some approximative heuristic algorithms can be found in, e.g., (Katayama and Narihisa, 2001), (Beasley, 1998), (Merz and Freisleben, 2002) and (Glover et al., 2002).

After a reformulation, several combinatorial optimization problems such as the maximum cut problem, the maximum clique problem, the maximum vertex packing problem and the maximum independent set problem can be written as BQP problems, (Merz and Freisleben, 2002). 
The results from the first part of the paper are then extended to an MIQP problem applicable to MPC problems with both binary and real-valued control variables.

This paper is organized as follows. In Section 2, the $\mathrm{BQP}$ and MIQP problems are defined. In Section 3 the preprocessing algorithm is derived for the $\mathrm{BQP}$ problem. A brief discussion of the implementation of the algorithm can be found in Section 4. Section 5 shows that the preprocessing algorithm can be extended to MIQP problems. In Section 6 the results are applied to the MPC problem. In Section 7, two examples of MPC are presented. Finally, section 8 contains conclusions and suggestions to future work.

\section{THE BQP AND MIQP PROBLEMS}

The MIQP problem can be seen as an ordinary QP problem for which some variables are constrained to be binary.

In this paper the problems considered are limited to those without any constraints except that some variables should be binary. We begin by considering a pure unconstrained $\mathrm{BQP}$ problem

$$
\mathscr{P}_{1}: \begin{cases}\min _{x} & x^{T} H x \\ \text { subject to } & x \in\{0,1\}^{n_{b}}\end{cases}
$$

where $H \in \mathbb{R}^{n_{b} \times n_{b}}$ is symmetric. The result is first extended to a BQP problem of the form

$$
\mathscr{P}_{2}: \begin{cases}\min _{x} & \frac{1}{2} x^{T} H x+f^{T} x \\ \text { subject to } & x \in\{0,1\}^{n_{b}}\end{cases}
$$

where we have incorporated a linear term in the objective function. Then, the result is extended to the unconstrained MIQP problem

$$
\mathscr{P}_{3}: \begin{cases}\min _{x} & \frac{1}{2} x^{T} H x+f^{T} x \\ \text { subject to } & x \in \mathbb{R}^{n_{c}} \times\{0,1\}^{n_{b}}\end{cases}
$$

The optimization vector $x$ contains $n_{c}$ real-valued and $n_{b}$ binary variables.

For what follows it is practical to define

$$
H=H_{d}+H^{+}+H^{-}
$$

where

$$
\begin{aligned}
H_{d, i j} & = \begin{cases}H_{i j}, & i=j \\
0, & i \neq j\end{cases} \\
H_{i j}^{+} & =\max \left(0, H_{i j}-H_{d, i j}\right) \\
H_{i j}^{-} & =\min \left(0, H_{i j}-H_{d, i j}\right)
\end{aligned}
$$

\section{PREPROCESSING FOR THE BQP PROBLEM}

To begin with, we consider the problem when all elements in $x$ are binary. The problem is then of the so-called BQP type, which is a pure combinatorial problem. As mentioned in Section 1, a characteristic property of combinatorial problems is that they in general are very hard to solve exactly, because of the computational complexity (Merz and Freisleben, 2002). To be able to solve large problem instances either an approximate algorithm or some special structure in the problem has to be used. If the structure in the problem is used it might be possible to solve the problems exactly in a reasonable time.

The algorithm presented in this section makes it possible to speed up the solution of a certain class of BQP problems. For this class of problems the algorithm produces an exact solution for one or more variables in polynomial time.

For each binary variable the algorithm delivers one out of three possible results: 1 is the optimal value, 0 is the optimal value or nothing can be said for sure.

The preprocessing algorithm is a consequence of the following theorem:

Theorem 1. For a BQP problem of type $\mathscr{P}_{1}$ the optimal value of one or more components $x_{i}$ can be found in polynomial time if for some $i \in\left\{1, . ., n_{b}\right\}$ any of the following conditions are fulfilled

$$
\left\{\begin{array}{l}
H_{i i} \geq-2 \sum_{j=1}^{n_{b}} H_{i j}^{-} \quad(i) \\
H_{i i}<-2 \sum_{j=1}^{n_{b}} H_{i j}^{+} \quad(i i)
\end{array}\right.
$$

If any of the conditions $(i)$ or $(i i)$ are fulfilled for a certain value of $i$, the optimal value of $x_{i}$ is given by

$$
x_{i}= \begin{cases}0, & \text { if (i) holds } \\ 1, & \text { if (ii) holds }\end{cases}
$$

PROOF. Consider the optimization problem $\mathscr{P}_{1}$, where $H$ is a symmetric $n_{b} \times n_{b}$ matrix. Denote the objective function by $Q(x)$ and rewrite it as follows

$$
Q(x)=x^{T} H x=\sum_{i=1}^{n_{b}} \sum_{j=1}^{n_{b}} H_{i j} x_{i} x_{j}
$$

where $x_{i}, x_{j} \in\{0,1\}, \forall i, j=\left\{1, \ldots, n_{b}\right\}$. For each $i \in$ $\left\{1, \ldots, n_{b}\right\}$ the objective function $Q(x)$ can be written on the form

$$
\begin{aligned}
Q(x) & =H_{i i} x_{i} x_{i}+2 x_{i} \sum_{j \neq i} H_{i j} x_{j} \\
& +g_{i}\left(x_{1}, x_{2}, \ldots, x_{i-1}, x_{i+1}, \ldots, x_{n_{b}}\right) \\
& =\left(H_{i i}+2 \sum_{j \neq i} H_{i j} x_{j}\right) x_{i} \\
& +g_{i}\left(x_{1}, x_{2}, \ldots, x_{i-1}, x_{i+1}, \ldots, x_{n_{b}}\right)
\end{aligned}
$$

where $g_{i}$ is a function that is independent of $x_{i}$ and the last equality follows from the fact that $x_{i}^{2}=x_{i}$ when $x_{i} \in\{0,1\}$.

Define

$$
h_{i}\left(x_{1}, x_{2}, \ldots, x_{i-1}, x_{i+1}, \ldots, x_{n_{b}}\right)=H_{i i}+2 \sum_{j \neq i} H_{i j} x_{j}
$$

Note that $h_{i}$ is independent of $x_{i}$. With this definition the objective function can be written as 


$$
\begin{aligned}
Q(x) & =h_{i}\left(x_{1}, x_{2}, \ldots, x_{i-1}, x_{i+1}, \ldots, x_{n_{b}}\right) x_{i} \\
& +g_{i}\left(x_{1}, x_{2}, \ldots, x_{i-1}, x_{i+1}, \ldots, x_{n_{b}}\right)
\end{aligned}
$$

Denote the optimal value of $x$ with $x^{*}=\left[x_{1}^{*}, \ldots, x_{n_{b}}^{*}\right]^{T}$. It is also convenient to make the following definitions

$$
\begin{aligned}
h_{i}^{*} & =h_{i}\left(x_{1}^{*}, x_{2}^{*}, \ldots, x_{i-1}^{*}, x_{i+1}^{*}, \ldots, x_{n_{b}}^{*}\right) \\
g_{i}^{*} & =g_{i}\left(x_{1}^{*}, x_{2}^{*}, \ldots, x_{i-1}^{*}, x_{i+1}^{*}, \ldots, x_{n_{b}}^{*}\right)
\end{aligned}
$$

It now follows that

$$
\min _{x} Q(x)=\min _{x_{i}} h_{i}^{*} x_{i}+g_{i}^{*}= \begin{cases}g_{i}^{*}, & \text { if } h_{i}^{*} \geq 0 \\ h_{i}^{*}+g_{i}^{*}, & \text { if } h_{i}^{*}<0\end{cases}
$$

From (11) the conclusion can be drawn that

$$
x_{i}^{*}= \begin{cases}0, & \text { if } h_{i}^{*} \geq 0 \\ 1, & \text { if } h_{i}^{*}<0\end{cases}
$$

Unfortunately, $h_{i}^{*}$ is usually not known before the optimal solution $\left\{x_{1}^{*}, x_{2}^{*}, \ldots, x_{i-1}^{*}, x_{i+1}^{*}, \ldots, x_{n_{b}}^{*}\right\}$ is known. A solution to this problem is to try to make an estimate of $h_{i}^{*}$. To simplify the notation, define $\bar{h}_{i}=\max _{x} h_{i}$ and $\underline{h}_{i}=\min _{x} h_{i}$. It now holds that $\underline{h}_{i} \leq h_{i}^{*} \leq \bar{h}_{i}$. From this observation the following implications can be stated

$$
\begin{cases}\bar{h}_{i}<0 & \Rightarrow h_{i}^{*}<0 \\ \underline{h}_{i} \geq 0 & \Rightarrow h_{i}^{*} \geq 0\end{cases}
$$

By combining (12) and (13), the following conclusion can be drawn

$$
x_{i}^{*}= \begin{cases}0, & \underline{h}_{i} \geq 0 \\ 1, & \bar{h}_{i}<0\end{cases}
$$

From (5) and (8) it follows that

$$
\begin{aligned}
\bar{h}_{i} & =\max _{x} h_{i}=H_{i i}+2 \max _{x} \sum_{j \neq i} H_{i j} x_{j} \\
& =H_{i i}+2 \sum_{j=1}^{n_{b}} H_{i j}^{+}
\end{aligned}
$$

where in the last equality it has been used that it is the sign of $H_{i j}$ that determines whether the optimal value of $x_{j}$ is 0 or 1 . Analogously it follows that

$$
\underline{h}_{i}=\min _{x} h_{i}=H_{i i}+2 \sum_{j=1}^{n_{b}} H_{i j}^{-}
$$

Equation (14) can then finally be written on the desired form

$$
x_{i}^{*}=\left\{\begin{array}{l}
0, H_{i i} \geq-2 \sum_{j=1}^{n_{b}} H_{i j}^{-} \\
1, H_{i i}<-2 \sum_{j=1}^{n_{b}} H_{i j}^{+}
\end{array}\right.
$$

From (17) it is clear that the computational complexity is polynomial in the number of variables, i.e. in $n_{b}$.

Now the result is extended to problems of type $\mathscr{P}_{2}$.

Corollary 1. For a BQP problem of type $\mathscr{P}_{2}$ the optimal value of one or more components $x_{i}$ can be found in polynomial time if for some $i \in\left\{1, . ., n_{b}\right\}$ any of the following conditions are fulfilled

$$
\left\{\begin{array}{l}
H_{i i} \geq-2 f_{i}-2 \sum_{j=1}^{n_{b}} H_{i j}^{-} \\
H_{i i}<-2 f_{i}-2 \sum_{j=1}^{n_{b}} H_{i j}^{+}
\end{array}\right.
$$

If any of the conditions $(i)$ or $(i i)$ are fulfilled for a certain value of $i$, the optimal value of $x_{i}$ is given by

$$
x_{i}= \begin{cases}0, & \text { if (i) holds } \\ 1, & \text { if (ii) holds }\end{cases}
$$

PROOF. The result follows directly from Theorem 1 by recognizing the fact that

$$
Q(x)=\frac{1}{2} x^{T} H x+f^{T} x=x^{T}\left(\frac{H+2 \operatorname{diag}(f)}{2}\right) x
$$

for $x_{i} \in\{0,1\}$.

\section{IMPLEMENTATION}

The preprocessing algorithm can be implemented in several different ways. If the programming language supports matrix syntax, e.g. Matlab, the implementation can be seen as a straightforward implementation of Theorem 1 or Corollary 1. Using the pseudo code command RowSum and the relational operators $<$ and $\geq$, a quick sketch of the implementation is given by

$$
\begin{aligned}
& \operatorname{Row} \operatorname{Sum}\left(H_{d}+2 H^{+}\right)<0 \\
& \operatorname{RowSum}\left(H_{d}+2 H^{-}\right) \geq 0
\end{aligned}
$$

where RowSum returns the sum of each row of a matrix as a column vector and the relational operators are assumed to work componentwise, producing a binary vector as the result. In this way, Corollary 1 is applied to all indices $i$ simultaneously. If the optimal value is found for at least one component in $x$, it is possible to repeatedly apply the above mentioned test again to a smaller problem, excluding the indices for which optimal $x_{i}$ already have been found.

It is also possible to perform the tests in (19) one row at a time. Then, if an optimal value of a variable has been found for some index $i$, this value can be used to remove a column from $H$ in the remaining tests, assuming that the other columns of $H$ are modified appropriately. This results in a sharper test, since the already found optimal value is then no longer estimated by the worst case value.

After the algorithm has computed the optimal value of all components that it is capable of, an ordinary MIQP solver, or BQP solver, may be applied to compute the remaining ones.

\section{PREPROCESSING FOR THE MIQP PROBLEM}

If the $x$-vector is allowed to contain both real-valued and binary variables, the BQP problem becomes an MIQP problem. In this section, the problem is assumed to be of the type $\mathscr{P}_{3}$ and the $x$-vector is assumed to be of the form

$$
\begin{aligned}
x & =\left[\begin{array}{ll}
x_{c} & x_{b}
\end{array}\right]^{T} \\
x_{c} & \in \mathbb{R}^{n_{c}}, x_{b} \in\{0,1\}^{n_{b}}
\end{aligned}
$$


The objective function in $\mathscr{P}_{3}$ can be expressed as

$$
\frac{1}{2}\left[\begin{array}{ll}
x_{c}^{T} & x_{b}^{T}
\end{array}\right]\left[\begin{array}{ll}
H_{c c} & H_{c b} \\
H_{c b}^{T} & H_{b b}
\end{array}\right]\left[\begin{array}{l}
x_{c} \\
x_{b}
\end{array}\right]+\left[\begin{array}{ll}
f_{c}^{T} & f_{b}^{T}
\end{array}\right]\left[\begin{array}{l}
x_{c} \\
x_{b}
\end{array}\right]
$$

We now assume $H_{c c}$ to be positive definite. If all components in $x$ had been real-valued it would have been straightforward to use the first order necessary conditions for optimality to calculate an algebraic optimal solution to the problem. When some of the components in $x$ are binary this is no longer possible. However, the optimality conditions mentioned can anyway be used to express the optimal values of the real-valued variables as a function of the binary variables. The expression for the optimal real-valued variables is then given by

$$
x_{c}=-H_{c c}^{-1}\left(H_{c b} x_{b}+f_{c}\right)
$$

This expression is substituted into (21). The resulting optimization problem is a pure BQP problem. The objective function, disregarding constant terms, can be written as

$$
\begin{aligned}
\frac{1}{2} x_{b}^{T} \tilde{H} x_{b}+\tilde{f}^{T} x_{b} & \triangleq \frac{1}{2} x_{b}^{T}\left(H_{b b}-H_{c b}^{T} H_{c c}^{-T} H_{c b}\right) x_{b} \\
& +\left(f_{b}-H_{c b}^{T} H_{c c}^{-1} f_{c}\right)^{T} x_{b}
\end{aligned}
$$

In the calculations the symmetry of $H$ and $H^{-1}$ has been used. When the objective function is written on the form (23), Corollary 1 can be applied.

\section{APPLICATION OF THE RESULTS TO MPC}

The results from Section 5 is in this section applied to the MPC problem. It is desirable to find the control signal sequence which minimizes a certain criterion for a system on the form

$$
\begin{aligned}
x(k+1) & =A x(k)+B_{c} u_{c}(k)+B_{b} u_{b}(k) \\
z(k) & =M x(k)
\end{aligned}
$$

where $u_{c} \in \mathbb{R}^{n_{u c}}$ denotes real-valued control signals and $u_{b} \in\{0,1\}^{n_{u b}}$ denotes binary control signals. The signal $z \in \mathbb{R}^{n_{z}}$ denotes the control objective, i.e. the signal which is controlled by the reference signal.

In MPC, an optimization problem is solved at each sampling time $k$, based on the state $x(k)$. At time $k$ the optimization returns the optimal control signal sequence for all samples from time $k$ to time $k+$ $H_{p}-1$, where $H_{p}$ denotes the so-called prediction horizon. Although the optimal control signal sequence is known for $H_{p}$ samples, only $u(k)$ is actually applied to the system. In the next sample a new optimization is performed and the first component in the new optimal control signal sequence is applied, and so on. The criterion which is often minimized in MPC problems is for time $k$

$$
\begin{aligned}
& \sum_{j=0}^{H_{p}-1}\|z(k+j+1)-r(k+j+1)\|_{Q_{r}}^{2} \\
& +\left\|u_{c}(k+j)\right\|_{Q_{c}}^{2}+\left\|u_{b}(k+j)\right\|_{Q_{b}}^{2} \\
& =\left(\tilde{M}\left(H x(k)+S_{c} U_{c}+S_{b} U_{b}\right)-R\right)^{T} \times \tilde{Q}_{r} \\
& \times\left(\tilde{M}\left(H x(k)+S_{c} U_{c}+S_{b} U_{b}\right)-R\right) \\
& +U_{c}^{T} \tilde{Q}_{c} U_{c}+U_{b}^{T} \tilde{Q}_{b} U_{b} \\
& =\left(\alpha+\tilde{M} S_{b} U_{b}\right)^{T} \tilde{Q}_{r}\left(\alpha+\tilde{M} S_{b} U_{b}\right) \\
& +2 U_{c}^{T} S_{c}^{T} \tilde{M}^{T} \tilde{Q}_{r}\left(\alpha+\tilde{M} S_{b} U_{b}\right) \\
& +U_{c}^{T} S_{c}^{T} \tilde{M}^{T} \tilde{Q}_{r} \tilde{M} S_{c} U_{c}+U_{c}^{T} \tilde{Q}_{c} U_{c}+U_{b}^{T} \tilde{Q}_{b} U_{b}
\end{aligned}
$$

In the first equality, the definitions made in the Appendix have been used, and in the second equality the definition $\alpha=\tilde{M} H x(k)-R$ has been used.

Compared to MPC for real-valued control signals, the problem to minimize (25) is no longer a QP problem, since the control signals in $U_{b}$ are binary variables. Instead, the problem is an MIQP problem of the type $\mathscr{P}_{3}$. By ignoring constants and dividing the objective function (25) by two, it can be written on the form (21) with

$$
\begin{aligned}
x_{c} & =U_{c}, x_{b}=U_{b} \\
H_{c c} & =S_{c}^{T} \tilde{M}^{T} \tilde{Q}_{r} \tilde{M} S_{c}+\tilde{Q}_{c} \\
H_{c b} & =S_{c}^{T} \tilde{M}^{T} \tilde{Q}_{r} \tilde{M} S_{b} \\
H_{b b} & =S_{b}^{T} \tilde{M}^{T} \tilde{Q}_{r} \tilde{M} S_{b}+\tilde{Q}_{b} \\
f_{c} & =S_{c}^{T} \tilde{M}^{T} \tilde{Q}_{r} \alpha, f_{b}=S_{b}^{T} \tilde{M}^{T} \tilde{Q}_{r} \alpha
\end{aligned}
$$

The optimization problem can then of course also be expressed as a BQP problem on the form (23).

If the control signals are ordered according to their appearance in time, it can be seen that the $H$-matrix gets a structure where the envelope of the matrix elements descents horizontally and vertically away from the diagonal. How fast the modulus of the matrix elements descent seems to be dependent of the position of the poles of the controlled system. For example, stable real poles give a non-oscillating fade off while complex poles give an oscillating fade off. Unstable real poles do also give elements that fade out. This behavior has to be further investigated. This structure of the $H$-matrix in the MPC problem makes it easier to fulfill condition (i) or (ii) in Corollary 1.

\section{EXAMPLES}

In the following subsections, two examples are presented to which the preprocessing algorithm is applied.

In both examples, the problem has been solved using three different approaches and the corresponding computational times are presented in a table. In Approach I, an ordinary MIQP solver has been used. In Approach II, real-valued variables have been eliminated, as described in Section 5, before an ordinary MIQP solver has been used. No other preprocessing has been 
performed. By doing this reformulation of the problem, $n_{c}$ variables less have to be computed in each node in the branch and bound tree. This is not done completely for free. The expressions for $\tilde{H}$ and $\tilde{f}$ have to be calculated. In the examples, these calculations are included in the solution times presented. In a real world MPC problem, some of these calculations could probably have been re-used in several consecutive, or all, MPC optimizations. Finally, in Approach III realvalued variables first have been eliminated according to Section 5 and then the preprocessing algorithm has been applied to compute as many variables as possible. Generally, any variables then remaining are computed using an ordinary MIQP, or BQP, solver. In the examples in Sections 7.1 and 7.2, the preprocessing algorithm determines the optimal value of all variables. Therefore, the MIQP solver is actually never used in Approach III. In all three approaches a slightly modified version of the MIQP solver miqp.m presented in (Bemporad and Mignone, 2000) has been used. To be able to make a fair comparison, all available combinations of the settings "method" and "branchrule" in the solver have been tested. In the table, only the shortest solution time achieved is presented. For further information of available settings, see (Bemporad and Mignone, 2000).

As mentioned in Section 4, the preprocessing algorithm can be implemented in several different ways. In Matlab, an implementation with vectorized expressions has much better performance than one with forloops. The code in miqp. $m$ is not vectorized. Therefore, to be able to make a fair comparison between the MIQP solver and the preprocessing algorithm, the tests of the conditions from Corollary 1 in the preprocessing algorithm are performed one row at a time by using for-loops.

Both tests have been performed on a Sun UltraSPARCIIe $500 \mathrm{MHz}$ with $640 \mathrm{Mb}$ RAM running Sun OS 5.8 and Matlab version 6.5.1.

\subsection{Mass position control}

In this example, a mass is controlled in one dimension by two separate forces. One force is possible to control continuously and the other is applied binary with a certain magnitude and direction. The position of the mass is supposed to follow a reference signal which is $r(t)=10 \sin (t)$. The state $x_{1}$ is the velocity of the mass and the state $x_{2}$ is the position. The continuous state space description is given by

$$
\begin{aligned}
\dot{x} & =\left[\begin{array}{ll}
0 & 0 \\
1 & 0
\end{array}\right] x+\left[\begin{array}{cc}
1 & -5 \\
0 & 0
\end{array}\right]\left[\begin{array}{l}
u_{c} \\
u_{b}
\end{array}\right] \\
z & =\left[\begin{array}{ll}
0 & 1
\end{array}\right] x
\end{aligned}
$$

To obtain a system on the form (24), zero order hold sampling has been used with the sampling time 0.1 s. The problem is solved over a time horizon of 50 samples. The control signal cost is chosen in a way
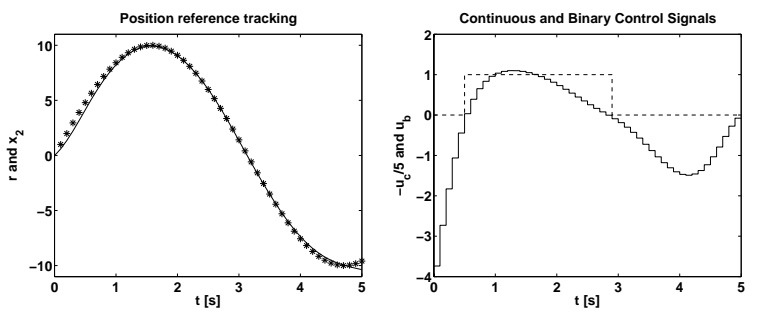

Fig. 1. The left plot shows how the position of the mass, $x_{2}$ (solid), follows the sampled reference signal, $r$ (starred), when the calculated optimal control signal sequence is applied to the continuous model of the system. The right plot shows the control signals, $u_{c}$ (solid) and $u_{b}$ (dashed). Note that $u_{c}$ is scaled in the plot.

that makes it beneficial to use the binary control signal, when it is possible. The cost function used in this example is of the type (25) with

$$
Q_{r}=100, Q_{c}=1, Q_{b}=1
$$

The result for initial state $x_{1}(0)=5$ and $x_{2}(0)=0$ is shown in Figure 1. The computational time for optimizing 50 real-valued and 50 binary variables is presented in Table 1. The tree exploring strategy used in the first and second approaches was the standard breadth first strategy. The node selection strategy was chosen to "max", see (Bemporad and Mignone, 2000). This combination of settings was the best choice available for this problem. In the third approach, all 50 binary variables were determined by the preprocessing algorithm based on Corollary 1. It can also be noticed that the computational time is reduced with a factor of about 180 .

Table 1. Performance tests

\begin{tabular}{lc} 
Optimization method & Solution time [s] \\
\hline Approach I & 15.861 \\
Approach II & 4.618 \\
Approach III & 0.0868
\end{tabular}

\subsection{Satellite attitude control}

In this example the optimal control signal sequence for the attitude control of a satellite is calculated. The satellite controls its attitude by accelerating a reaction wheel inside the satellite and by using external thrusters. When the wheel is accelerated a counter torque is produced. If several adjustments in the same direction are made, the angular velocity of the wheel finally becomes very high, or at least high enough to be power consuming in steady state. To be able to slow down the wheel without affecting the attitude of the satellite, the external thrusters have to be used to compensate when the wheel is braked.

The wheel is assumed to be controlled continuously by an electric engine. Its control signal is denoted $u_{c}$. The satellite is also assumed to be equipped with two external thrusters, one in each direction. These are assumed to be controlled binary, i.e. either they give 

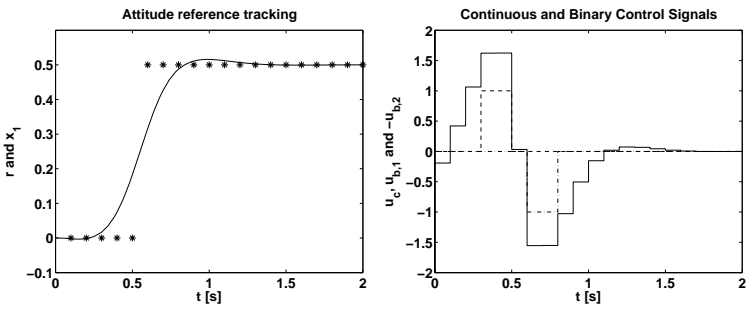

Fig. 2. The left plot shows how the attitude of the satellite, $x_{1}$ (solid), follows the sampled reference signal, $r$ (starred), when the calculated optimal control signal sequence is applied to the continuous model of the system. The right plot shows the control signals, $u_{c}$ (solid), $u_{b, 1}$ (dashed) and $u_{b, 2}$ (dash-dotted).

full thrust or no thrust at all. The binary control signals for the thrusters are denoted $u_{b, 1}$ and $u_{b, 2}$.

A continuous time state space description for the system with satellite attitude $x_{1}$, satellite angular velocity $x_{2}$ and internal wheel velocity $x_{3}$ is

$$
\begin{aligned}
& \dot{x}=\left[\begin{array}{lll}
0 & 1 & 0 \\
0 & 0 & 0 \\
0 & 0 & 0
\end{array}\right] x+\left[\begin{array}{ccc}
0 & 0 & 0 \\
2.5 & 1 & -1 \\
-10 & 0 & 0
\end{array}\right]\left[\begin{array}{c}
u_{c} \\
u_{b, 1} \\
u_{b, 2}
\end{array}\right] \\
& z=I_{3} x
\end{aligned}
$$

To obtain a system on the form (24), zero order hold sampling with the sampling time $0.1 \mathrm{~s}$ has been used. The time horizon used is 20 samples. The cost function used in this example is of the type (25) with

$$
\begin{aligned}
& Q_{r}=\operatorname{diag}\left(0.5 \cdot 10^{4}, 10^{-2}, 10^{-1}\right) \\
& Q_{c}=10, Q_{b}=10 \cdot I_{2}
\end{aligned}
$$

In the simulation, the initial state was chosen to $x_{1}(0)=0, x_{2}(0)=0$ and $x_{3}(0)=0$. In this example, the reference signal for the attitude of the satellite is a step function with the amplitude 0.5. The optimal control signal sequence and the attitude of the satellite is shown in Figure 2. The computational time for optimizing 20 real-valued and 40 binary variables is found in Table 2. The tree exploring strategy used in the first and second approaches was ordinary depth first, and the node selection strategy was chosen to "min", see (Bemporad and Mignone, 2000). This combination of settings was the best choice available for this problem. In this example the preprocessing algorithm determined 40 out of 40 binary variables. The computational time was reduced with a factor of about 275 .

Table 2. Performance tests

\begin{tabular}{lc} 
Optimization method & Solution time [s] \\
\hline Approach I & 11.449 \\
Approach II & 6.916 \\
Approach III & 0.0414
\end{tabular}

\section{CONCLUSIONS}

In this paper a preprocessing algorithm for MIQP and $\mathrm{BQP}$ problems has been derived. These problems are generally known to have exponential complexity. Given a problem with the desired properties, the preprocessing algorithm can compute the optimal values of some, or all, of the optimization variables in polynomial time. For certain examples, it has been shown that the preprocessing algorithm can reduce the computational time significantly. In some cases the computational time was reduced with a factor of 275 . A suggestion to future work is to find more applications where the preprocessing algorithm can be applied.

\section{REFERENCES}

Beasley, J. E. (1998). Heuristic algorithms for the unconstrained binary quadratic programming problem. Technical report. Management School, Imperial College, UK.

Bemporad, A. and D. Mignone (2000). A Matlab function for solving Mixed Integer Quadratic Programs Version 1.02 user Guide. Institut für Automatik, ETH.

Bemporad, A. and M. Morari (1999). Control of systems integrating logic, dynamics and constraints. Automatica 35(3), 407-427.

Bemporad, A., D. Mignone and M. Morari (1999). An efficient branch and bound algorithm for state estimation and control of hybrid systems. In: Proceedings of the European Control Conference.

Garey, M. and D. Johnson (1979). Computers and Intractability: A guide to the Theory of NP-Completeness. Freeman.

Glover, F., B. Alidaee, C. Rego and G. Kochenberger (2002). One-pass heuristics for large-scale unconstrained binary quadratic problems. European Journal of Operational Research 137(2), 272-287.

Katayama, K. and H. Narihisa (2001). Performance of simulated annealing-based heuristic for the unconstrained binary quadratic programming problem. European Journal of Operational Research 134(1), 103-119.

Merz, P. and B. Freisleben (2002). Greedy and local search heuristics for unconstrained binary quadratic programming. Journal of Heuristics 8(2), 197-213.

Savelsbergh, M. (1994). Preprocessing and probing techniques for mixed integer programming problems. ORSA Journal on Computing.

\section{APPENDIX}

In this appendix the definitions of the vectors and matrices used when the objective function for the MPC problem is cast on the form (25) are presented. The system to be controlled is assumed to be on the form (24).

$$
\begin{aligned}
& U_{c}=\left[u_{c}(k) u_{c}(k+1) \ldots u_{c}\left(k+H_{p}-1\right)\right]^{T}, \\
& U_{b}=\left[u_{b}(k) u_{b}(k+1) \ldots u_{b}\left(k+H_{p}-1\right)\right]^{T} \text {, } \\
& R=\left[r(k+1) r(k+2) \ldots r\left(k+H_{p}\right)\right]^{T}, \\
& H=\left[\begin{array}{llll}
A & A^{2} & \ldots & A^{H_{p}}
\end{array}\right]^{T} \text {, } \\
& S_{c}=S\left(B_{c}\right), S_{b}=S\left(B_{b}\right) \text {, } \\
& S\left(B_{*}\right)=\left[\begin{array}{cccc}
B_{*} & 0 & \ldots & 0 \\
A B_{*} & B_{*} & \ldots & 0 \\
\vdots & \vdots & \ddots & \vdots \\
A^{H_{p}-1} B_{*} & A^{H_{p}-2} B_{*} & \ldots & B_{*}
\end{array}\right] \text {, } \\
& \tilde{M}=\operatorname{blkdiag}(M, \ldots, M), \tilde{Q}_{r}=\operatorname{blkdiag}\left(Q_{r}, \ldots, Q_{r}\right) \text {, } \\
& \tilde{Q}_{c}=\operatorname{blkdiag}\left(Q_{c}, \ldots, Q_{c}\right), \tilde{Q}_{b}=\operatorname{blkdiag}\left(Q_{b}, \ldots, Q_{b}\right) \text {. }
\end{aligned}
$$

